
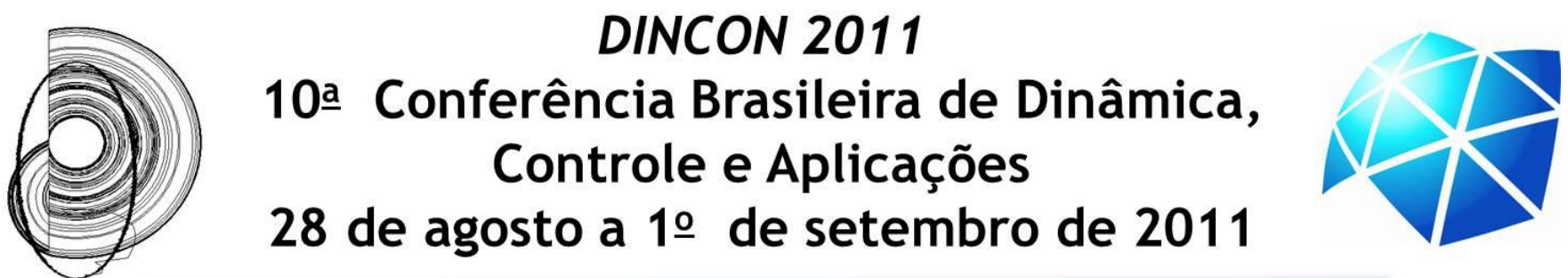

\title{
EMPIRICAL ANALYSIS OF A NEW WINDING END TURN CROSS-SECTION FOR ELECTRIC MOTORS
}

\author{
Sherfis Gibran Ruwer, Mário César Ricci \\ Instituto Nacional de Pesquisas Espaciais - INPE, São José dos Campos, Brasil \\ sherfis@yahoo.com.br,mcr@dem.inpe.br
}

\begin{abstract}
Some engineering applications require an efficient and light electric motor. The end turn of motor windings are analyzed from a weight aspect to minimize material usage. An empirical study was done to develop an end turn geometry focused in the minimization of its volume.
\end{abstract}

Keywords: winding end turn, electric motor, engineering applications.

\section{INTRODUCTION}

There are applications where a reduced size and light weight electric motor should be required. This article presents an empirical study of the end turn geometry for windings production. The focus was reduces the volume of the end turns. This ensures minimal material and less electromagnetic loses due to current flux in the end turn.

This work is a section of a whole, where an optimized electric motor should be developed in accordance with high efficiency and little weight. The first step was study the newest techniques of winding end turn forming and evaluate a model empirically with upgrades. Next, it will be developed a mathematical model for optimize the volume with better known constraints, some of them raised from this first analysis.

The design was done for the windings of an ironless, brushless and coreless electric motor with radial magnetic flux. It has permanent magnets which are interposed externally and internally with respect to the winding stator. The stator was build molding a set of wave shaped windings self-supported by a resin compressed through it.

The main problem to solve in the winding shape for the stator is the minimization of the winding overall volume with the maximization of the amount of conductor material related to the space filled with resin. In this way the problem can be reduced to the minimization of the relation of the conductor material volume in the end turns of the winding and the overall winding volume. This is because the active portions of winding, called legs, which produce the magnetic torque, have a rectangular shape with rounded corners and can be easily distributed over the winding circumference. Thus, one can minimize the end turns, what do not produce torque for the motor.

\section{METHODS}

Even though this article do not use mathematical models to prove the development, it was applied the experience of the authors with empirical analysis. The design was managed into an iterated and incremented cycle method. At each step of the iteration procedure a document with the increments - the upgrades done at that cycle - were written. A new tridimensional model was developed when the analysis were done. Thus, the problems could be detected and solutions could be defined, or mainly new suggestions of improvement were achieved.

After sixteen cycles it was completed a document with the difficulties encountered to minimize the end turns and a list of improvements defined. Based on that register, the next work will be based on to build a mathematical model of the tridimensional geometry for the winding end turn. At the following will be described the main steps about the development of the sixteen models.

\subsection{The First Model}

As explained, the motor studied was ironless, coreless and brushless, with radial magnetic flux. Their windings were wave formed. As shown in Fig. 1 the problem is increase the cross section of the coils at the same time the volume of the end turns should be minimized. This is done because the end turns do not contribute to the magnetic flux used to produce the motor torque. Also, the material in the end turns can be reduced, decreasing the motor's weight and the electromagnetic loses in the conductors.

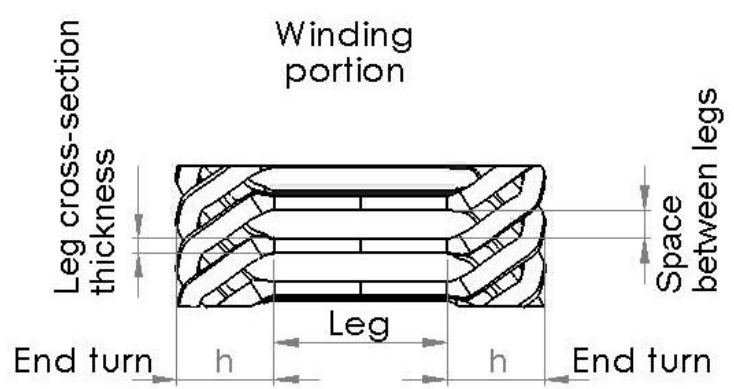

Fig. 1. Winding minimization problem 
The models were drawn in mechanical CAD. The first model, in Fig. 2 presents a thin cross section of electric conductor. That dimension is limited due to the end turn geometry. As three coils are packaged in sequence, as in Fig. 1, one should counteract the height - dimension $h$ - of the end turn and the thickness of its cross section. It is important to point that, even the end turns do not produce magnetic torque for the motor its cross-section should be kept the same of the legs.

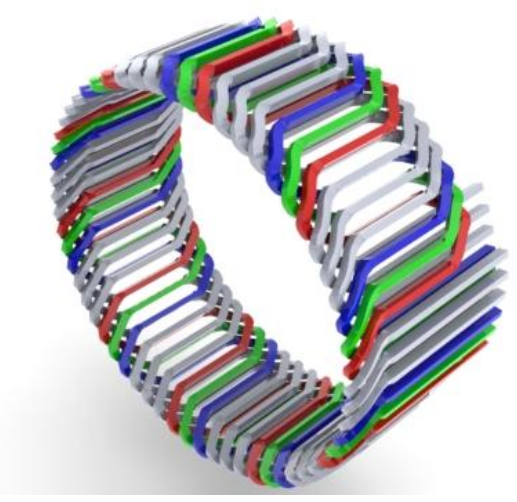

(a) First stator - with much space between the winding legs

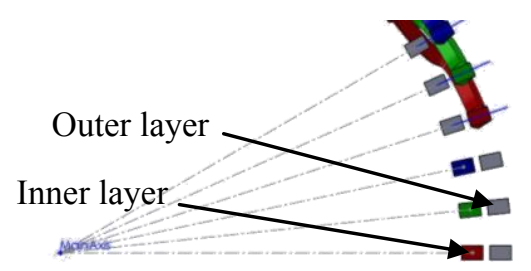

(b) Cross section of the winding legs.

Fig. 2. First tridimensional model

In the first model, Figure 2, the end turn path was projected as a straight path from the outer leg to a point centered between that leg and the next one, positioned in the inner layer. At that point the path was curved radial to the inner layer, and after it follows a straight line to the inner leg. In a good arrangement of the end turn path and the end turn cross section, it was reached a bad relation between the leg cross-section thickness and the space between them.

\subsection{Incremental Models}

The second end turn model was developed based on a helical tridimensional path between the adjacent legs. The cross section was an ellipse. The helical path had 180 degrees over a centered path between the inner and the outer legs cylindrical layers. The model shows that the end turn cross section area could not achieve the leg cross section area without overlap the adjacent end turn. This means it should have more space to the end turn path but less space compared to the first model.

Then the space of the end turn was increased in the helical radial direction, what means the end turn helical radius should exceed the inner-outer layer distance. To mount this stator in the motor, the helical axis should be translated a small distance in the direction to the center of the stator in one side of the legs, and to the radial (external) direction in the other side of the legs. Thus, the end turns enable the stator to be mounted in the inner set of magnets, and after the outer set of magnets could be mounted around the stator, with a minimum distance between the magnets and the active portion of the stator. This means the helical axis should be shifted from the middle between the two adjacent legs. After the model was built, it was suggested to increase the helical angle from 180 to 225 degrees to improve the connection shape between the end turn and both legs. This model was done with an elliptical end turn cross section as shows Fig. 3.

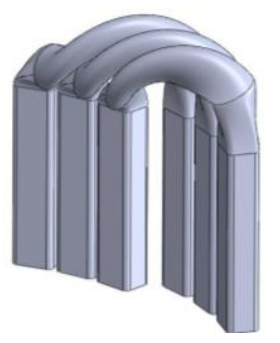

Fig. 3. Model with helical path and elliptical end turn cross section

It seems to enable a shorter distance between the legs. It then increases the stator relation between the legs cross section thickness and the space among them. After some analysis of this model, pointed a cross section view of the stator with a plane through the stator axis, it was identified that the elliptical cross section of the end turns leaved an empty space in the central axis of the helical path. If the helical radius was reduced, the cross section of the adjacent end turns overlaps. At the same time the outer portion of the ellipse leave empty spaces. With this analysis a new shaped end turn cross section was invented: a "drop" shape. As the end turn path rotates as a helical, the inner portion of the cross section should have less material and the outer portion could have more. Looking through the helical axis the thickness of the cross section should increase as the radius of the helical increase, forming a "drop" shape. The drop shaped cross section, in Fig. 4, should have its corner rounded. This new model increases the fill factor.

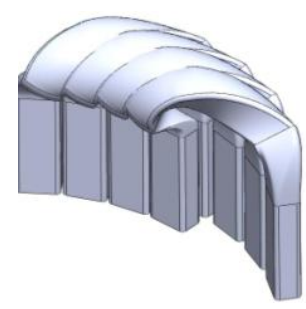

(a) Portion of the stator

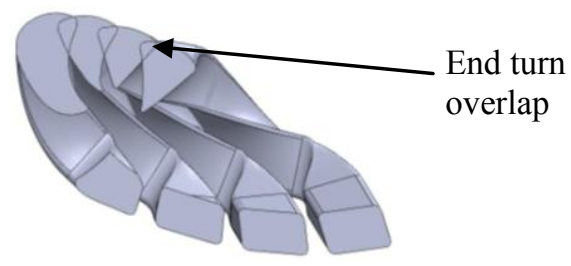

(b) A portion of the stator in cross-section view - drop shape

Fig. 4. End turn with cross section in drop shape 

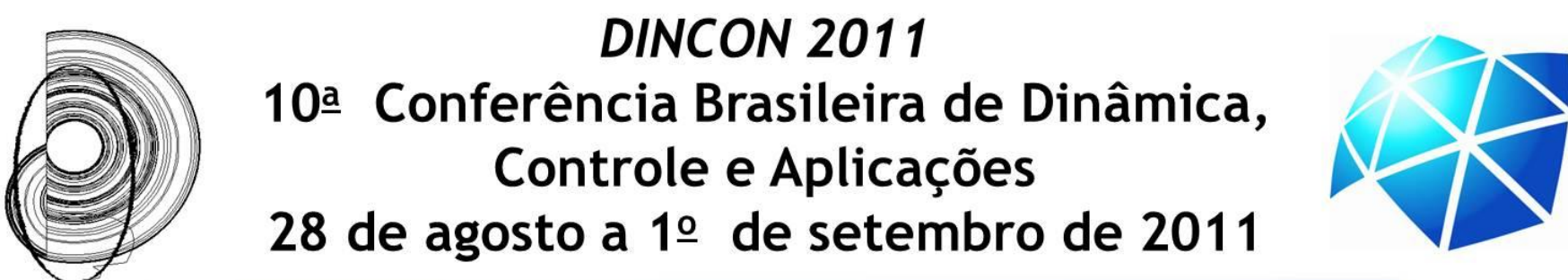

But it was identified that also the main stator radius implies the same relation as above. The inner portion of the end turn, meaning the portion near the inner cylindrical layer, should be thicker then the outer. One solution, presented in Fig. 5, developed was rotates the cross section along the path. The cross section major thickness at the beginning of the end turn helical path, located at the outer leg, is approximately aligned to the tangent of the outer cylindrical stator layer. It is rotated continuously to achieve the end of the path aligned radial in the stator. This way, the cross section major thickness have space in the tangent of the outer cylindrical layer to minimize the empty space between each two adjacent end turns, at the same time the inner portion can state with minimum space between each end turn in the inner radius of the stator because the tangent thickness of this cross section was the smaller one. This last arrangement optimizes the end turn space filled with copper.

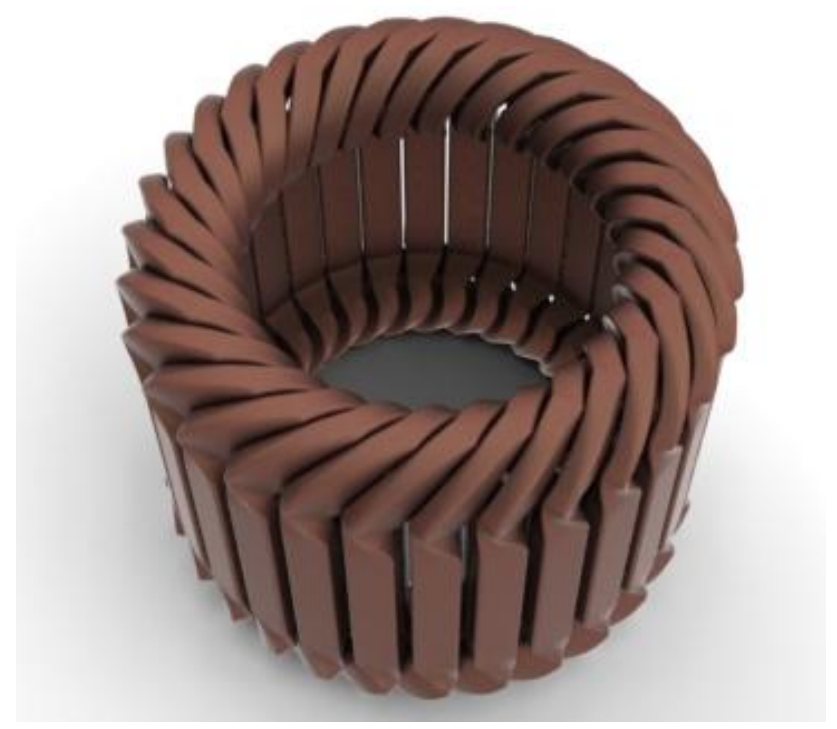

Fig. 5. End turn with helical path and cross section rotated along the path.

\section{RESULTS}

Comparing the first model (Fig. 1) with the last (Fig. 5) one can see that the space between each two legs in the first is greater than the space in the last. As results, the main parameters for a future mathematical model of the end turn geometry were defined. They are explained in the next section.

In this study the focus is in the side of the winding where the end turn is flatted to the inner direction, what is the most difficult to accommodate the end turns due to the little radius compared to the other side. This way, the side where the end turn is flatted to the outer direction was not modeled here.

\section{CONCLUSION}

A first cycle of incremental design shows the main parameters for an end turn model with optimized geometry. These parameters will be deal in the next study. They will be introduced to mathematical models of the end turn geometry. The mathematical geometry will be studied to find a minimized volume that achieves a specified crosssection area of the conductor. The next paragraphs explain the parameters raised from the present analysis.

The crucial side to be defined is the one inner flatted, to the direction of the main axis of the stator. This side has less size to develop the end turn, and so it should be optimized to have the same cross-section of the legs but in a minimized volume.

Viewing the end turn as Fig. $6 a$ it can be found that due to the radius $R_{h}$ of the helical the inner portion of the helical has less space, what means the design should decrease the material in the inner radius, and increase the thickness of the end turn curve in the outer side of $R_{h}$.

In Fig. $6 b$, the same analysis is done in $R_{s}$, the stator main radius. The portion of the end turn curve near the center of the stator, for $R_{s}$ small, has less space to be designed, thus its cross-section, in an quasi-elliptical shape, can be rotated aligning its major axis with $R_{s}$. Also, the other side, for $R_{s}$ large, can have their major axis perpendicular to $R_{s}$.
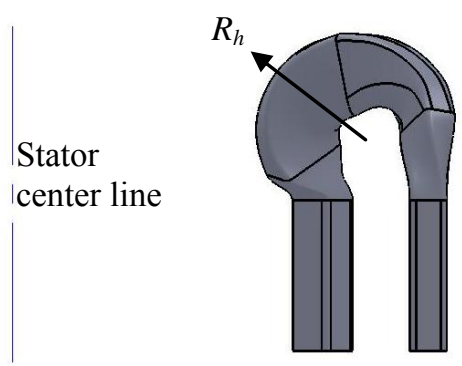

(a) End turn detail - radial view

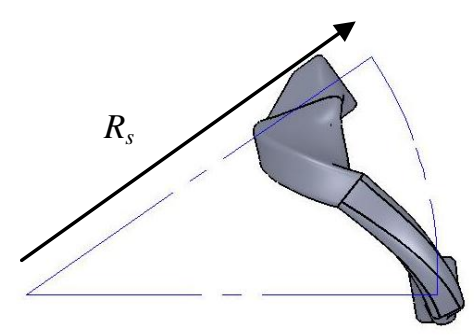

(b) End turn detail - top view

Fig. 6 - Last model details 